March 13, 2022 5:0 WSPC/INSTRUCTION FILE hepro4'lenain

International Journal of Modern Physics: Conference Series

(C) World Scientific Publishing Company

\title{
THE H.E.S.S. EXTRAGALACTIC SKY
}

\author{
JEAN-PHILIPPE LENAIN, FOR THE H.E.S.S. COLLABORATION \\ LPNHE, Université Pierre et Marie Curie Paris 6, Université Denis Diderot Paris 7, \\ CNRS/IN2P3, 4 Place Jussieu \\ 75252 Paris Cedex 5, France. \\ jlenain@lpnhe.in2p3.fr
}

Received Day Month Year

Revised Day Month Year

\begin{abstract}
More than fifty extragalactic very high energy (VHE; $E>100 \mathrm{GeV}$ ) sources have been found using ground-based imaging atmospheric Cherenkov telescopes, about twenty of which have been discovered using the H.E.S.S. (High Energy Stereoscopic System) experiment based in Namibia. Even though BL Lac objects are the dominant class of VHE detected extragalactic objects, other types of sources (starburst galaxies, radio galaxies or flat spectrum radio quasars) begin to emerge. A review of the extragalactic sources studied with H.E.S.S. is given, with an emphasis on new results.
\end{abstract}

Keywords: Gamma rays: astronomical observations; Blazars; Radio galaxies; Clusters: galaxy; Gamma rays: bursts; Cosmic background radiation.

PACS numbers: 95.85.Pw, 98.54.Cm, 98.54.Gr, 98.65.-r, 98.70.Rz, 98.70.Vc

\section{The H.E.S.S. Experiment}

The H.E.S.S. experiment has been used for more than ten years to explore the very high energy (VHE; $E>100 \mathrm{GeV}$ ) sky in the Southern hemisphere with four imaging atmospheric Cherenkov telescopes (IACT) operated in Namibia. In September 2012, a fifth telescope located in the middle of the original array was inaugurated (see Fig. 11. Its mirror surface of $600 \mathrm{~m}^{2}$ will enable a notable gain in sensitivity as well as a lower energy threshold of a few tens of GeV.

H.E.S.S. is used to study different physics topics, such as the origin of Galactic cosmic rays, the acceleration processes at work in compact objects, or searching for indirect signs of dark matter in the Universe. Observations of many types of objects are thus performed with H.E.S.S., from supernova remnants to galaxy clusters, pulsar wind nebulæ or active galactic nuclei (AGN). This manuscript focuses on recent results obtained with H.E.S.S. on extragalactic sources.

The number of known very high energy sources has dramatically increased with the advent of the current generation of IACT, with now almost 150 detected objects. At the time of writing this manuscript, from the 58 detected extragalactic VHE 


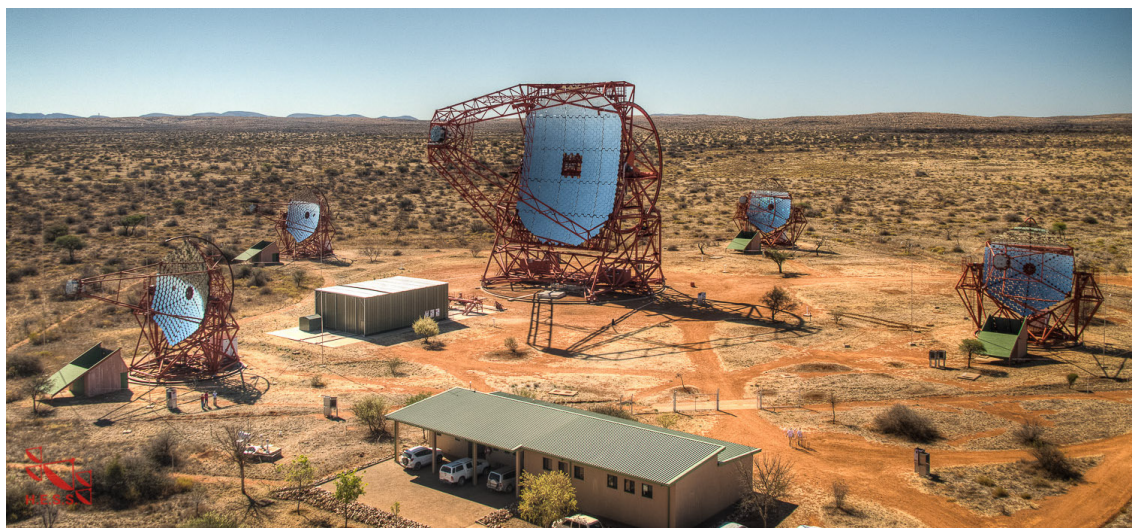

Fig. 1. The H.E.S.S. array of five imaging atmospheric Cherenkov telescopes in Namibia.

emitters, 52 objects are of the blazar type with 49 BL Lac object: $\rrbracket^{\circledR}$

\section{Recent H.E.S.S. Results}

\subsection{Starburst galaxies}

Following deep observations of more than $170 \mathrm{~h}$ of good quality data, the starburst galaxy NGC 253 was detected at a $7.1 \sigma$ confidence level (CL) and a spectrum with a photon index of $\Gamma=2.14 \pm 0.18_{\text {stat }}$ was derived ${ }^{11}$ Detailed analysis of both H.E.S.S. and public Fermi/LAT data revealed a joint spectrum with a photon index of $\Gamma=2.34 \pm 0.03$ in the high and very high energy ranges (see Fig. 2). Studying this high energy emission, compared to theoretical models, the $\gamma$-ray emission is likely to be dominated by hadronic interactions within the starburst region. More than $20 \%$ of the non-thermal energy is converted into $\pi^{0} \gamma$-ray production, assuming an efficiency of $10 \%$ for cosmic ray acceleration processes from supernova remnants in the starburst region.

\subsection{Radio galaxies}

Up to now, three radio galaxies have been detected in the VHE domain, namely NGC $1275, \frac{[2]}{M} 87^{3} \sqrt[4]{4}$ and Cen ${ }^{5,5}$ all of them belonging to the FR-I type. The VHE emitting source IC 310 also used to be classified as a head-tail radio galaxy, even though recent studies have revealed blazar-like properties 6.67

More specifically, the radio galaxy M 87 has been observed for more than 10 years at high energies, along with extended multi-wavelength campaigns in the $\mathrm{X}$ -

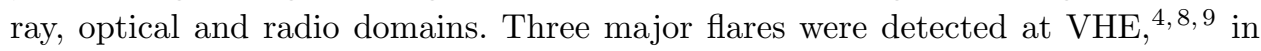
2005, 2008 and 2010. However, no unique multi-wavelength pattern was identified in this object. Indeed the question of the location of the emission zone arose when the

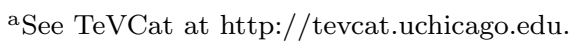




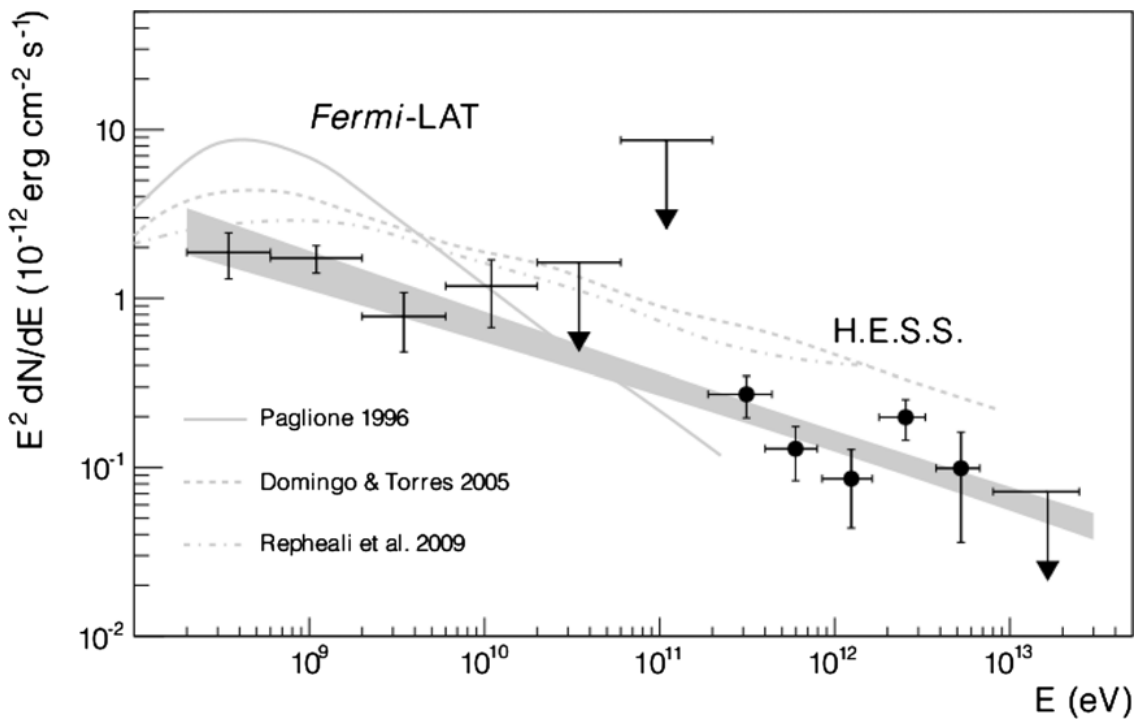

Fig. 2. Spectral energy distribution of NGC 253 observed with H.E.S.S. and Fermi/LAT, with data fit simultaneously in shaded area ( $1 \sigma$ confidence band) and theoretical predictions. See Ref. 1 for more details.

2005 flare showed multi-wavelength correlation between observations in VHE and Xray ranges from the knot $H S T-1$, while the temporal analysis of the two other flares seemed to establish the central part of the jet as the high energy emitter ${ }^{8}[9]$ In 2010 , the flare profile was unambiguously observed as asymmetrical, with a characteristic variability timescale of the order of one day. In radio, VLBA $43 \mathrm{GHz}$ observations show no indication for an enhanced flux from the base of the jet in the 2010 event, contrasting with radio observations of the 2008 flare for which a radio burst was detected contemporaneously with VHE $\gamma$-ray emission. The complex variability behaviour of this source particularly strengthens the case for strictly simultaneous multi-wavelength observations to help pinpointing the emission processes at work in high energy emitting objects.

Cen A, the closest FR I radio galaxy, has been detected at VHE with H.E.S.S! 5 using more than $120 \mathrm{~h}$ of observations. The Fermi/LAT collaboration reported the detections of its giant radio lobes $\frac{10}{10}$ as well as central AGN activity at high energies ${ }^{11}$ Refs. 5 and 11 have shown that the high and VHE spectra are only marginally compatible, and simple one-zone synchrotron self-Compton (SSC) modelling applied to this object requires an unusually low Doppler factor, with respect to BL Lac objects, due to the large angle of the jet axis to the line of sight ${ }^{11}$ These facts support the need for more elaborate interpretations, such as multi-zone models, $12 \mid 13$ decelerating flows ${ }^{14}$ or high energy emission processes in the vicinity of the central black hole magnetosphere ${ }^{[15}$ More recently, a potential additional hard spectral component was found in Fermi/LAT data, ${ }^{16}$ emerging at around $4 \mathrm{GeV}$ and reconciling the marginal mismatch between Fermi/LAT and H.E.S.S. data, further supporting 
the presence of multiple emitting components. The observations of time variability at high energies, not detected so far, would definitely help in distinguishing between the currently viable scenarios to explain this complex source.

\subsection{Gamma-ray bursts}

Gamma-ray bursts show evidence of strongly enhanced relativistic outflows and are established as powerful particle accelerators up to the high energy domain! 17 However, so far no $\gamma$-ray burst has been detected in the VHE domain, even though extensive search has been followed on this path. Both the enhanced sensitivity and probed energy range with the future Cherenkov Telescope Array (CTA) promise the detection of about a few events per year $18 \mid 19$

Serendipitous observations of GRB 060602B were made with the H.E.S.S. array, when an alert was received for an event occurring in the field of view of H.E.S.S. observations at that time, resulting in observations of both the prompt and afterglow phases at VHE. 20 The H.E.S.S. observations did not unveil any significant VHE $\gamma$ ray signal, but the very soft Swift/BAT spectrum and its proximity to the Galactic centre led Ref. 21 to favor a Galactic X-ray burster origin for this peculiar event, instead of a cosmological one.

\subsection{Galaxy clusters}

Powerful active galactic nuclei can be found at the centre of some galaxy clusters, whose outbursts generate feedback reactions in the embedding intra-cluster medium. Such interactions can be detected for instance by the presence of cavities in the Xray emission. ${ }^{22}$ Cosmic rays represent a viable solution to explain the main pressure support of such bubbles. In this case, $\gamma$-ray emission is expected in these objects: inelastic collisions of cosmic rays with the thermal ambient medium would result in VHE $\gamma$-rays from hadronic processes, while electronic cosmic rays would inverse Compton up-scatter with the cosmic microwave background or the extragalactic background light.

Hydra A is the closest galaxy cluster hosting such an AGN activity. The Chan$d r a$ satellite revealed a large scale cavity built up from three generations of AGN outbursts 5 Both giant outer lobes, dominating the system energetics, and inner lobes, filled with the youngest population of particles, are of interest for the search for $\gamma$-ray emission. Fermi /LAT and H.E.S.S. observations of Hydra A resulted in no detection in the high and very high energy domains. ${ }^{24}$ However, the upper limits derived on the $\gamma$-ray flux help constraining theoretical models: for the outer giant lobes, in which hadronic processes dominate, a full mixing of cosmic rays within the intra-cluster medium is excluded, with a degree of mixing less than 0.7 and 0.5 , respectively from H.E.S.S. and Fermi/LAT data. In the case of leptonic emission originating from the inner lobes, Fermi/LAT and $X M M$ data tend to favour a high magnetic field strength above $8 \mu \mathrm{G}$. 


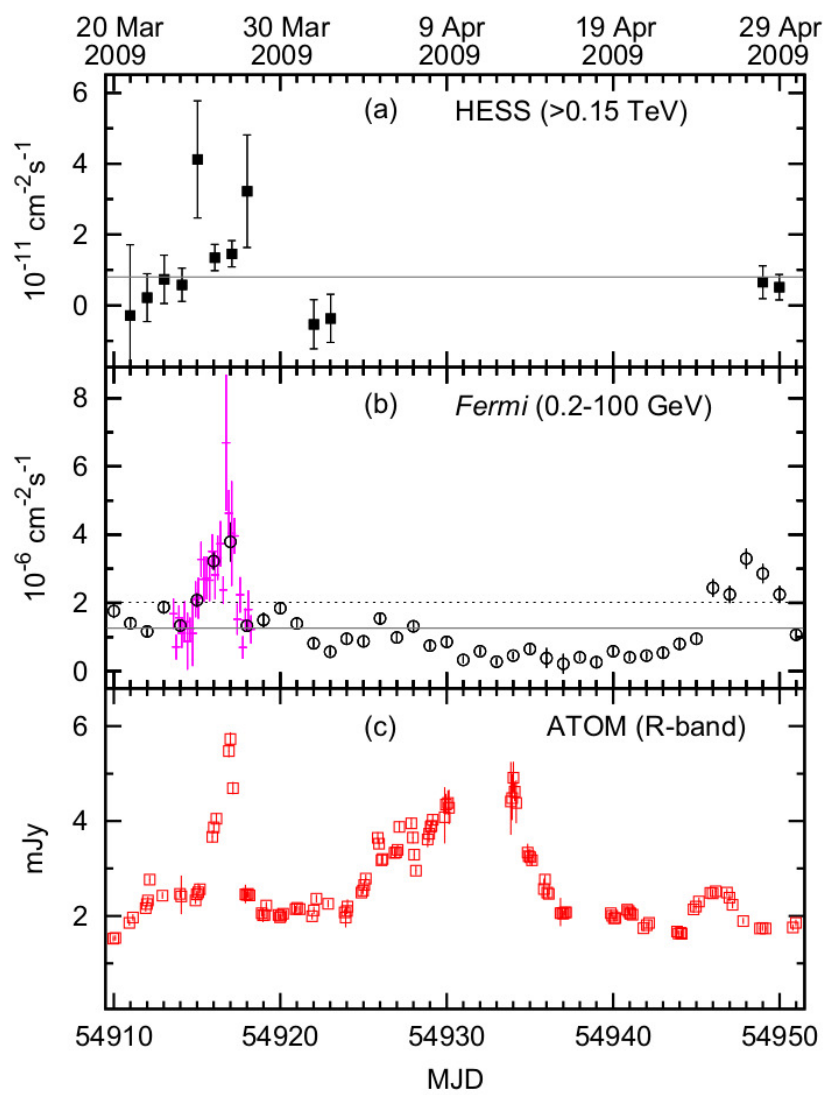

Fig. 3. Light curve of PKS 1510-089 in the VHE, high energy, and optical ranges. See Ref. 27 for more details.

\subsection{Flat spectrum radio quasars}

Due to the synchrotron peak of their emission lying at low frequency and their soft $\gamma$-ray spectrum, flat spectrum radio quasars (FSRQ) are rare at VHE, with only three known sources: $4 \mathrm{C}+21.35, \frac{25}{2} 3 \mathrm{C} 27 \mathrm{9}^{26}$ and PKS $1510-089.27$ The later was detected at a $9.2 \sigma \mathrm{CL}$ in about $16 \mathrm{~h}$ of good quality H.E.S.S. data. Even though no significant variability was detected in the VHE range, contemporaneous observations with Fermi/LAT in the high energies and ATOM in the optical band revealed strong variability on a day scale (see Fig. 3). The VHE spectrum was used to derive upper limits on the photon density of the extragalactic background light (EBL, see Section 2.7). For this particularly far - for VHE standards - object $(z=0.361)$, these limits were found to be compatible to those derived from relatively nearby BL Lac objects. 28 


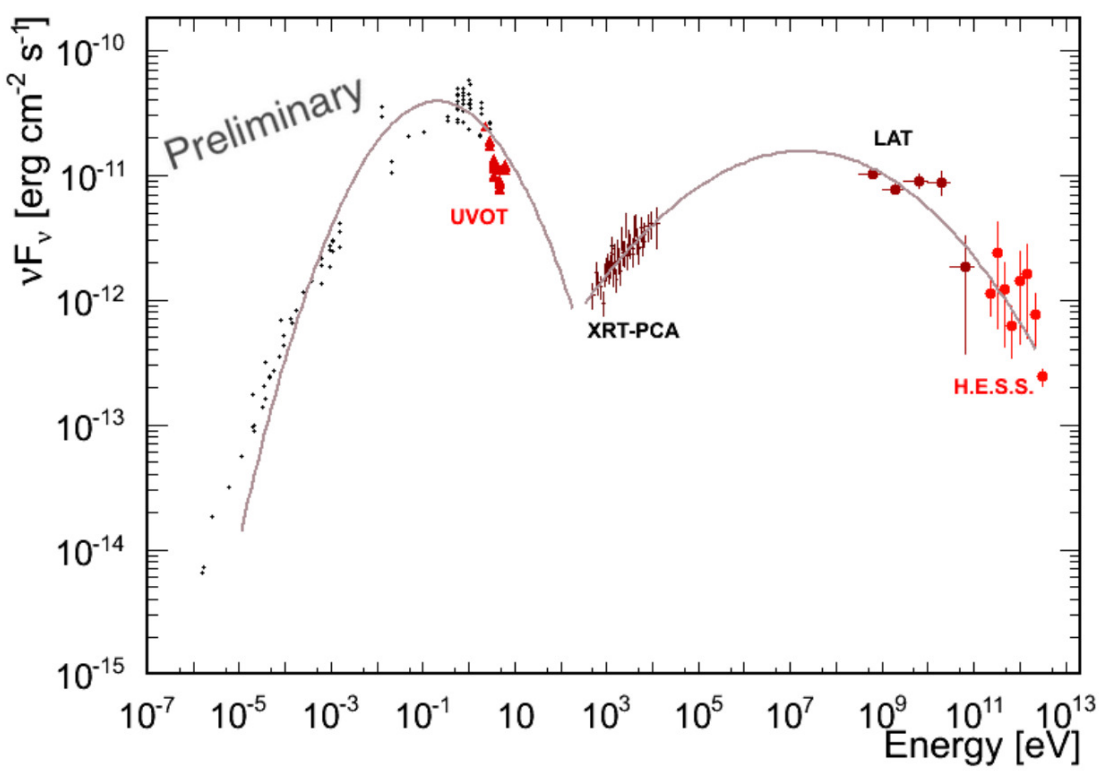

Fig. 4. Spectral energy distribution of AP Lib. See Ref. 36 for more details.

\subsection{BL Lac objects}

BL Lac objects are by far the most numerous known extragalactic VHE emitters. This can be explained by the fact that their jet, pointing close to the observer's line of sight, Doppler boost their broadband emission, with the higher frequency-peaked objects being natural candidates for $\gamma$-ray emission.29

Among these, the high-frequency-peaked BL Lac object (HBL) PKS 0447-439 was discovered at VHE with H.E.S.S. at the $15.2 \sigma$ CL in $13.5 \mathrm{~h}$ of observations ${ }^{30}$ Multi-wavelength observations with Fermi/LAT, Swift, ATOM and ROTSE were used to draw a contemporaneous spectral energy distribution of this source, which was modelled using an SSC model. The redshift of this object being uncertain $\sqrt[31]{32} 33 \sqrt[34]{34}$ the modelling of multi-wavelength data was used to derive an upper limit on its redshift of $z<0.59$.

1ES $1312-423$ is an HBL which was serendipitously discovered ${ }^{\sqrt{35}}$ with H.E.S.S. in the field of view of observations of Cen A. Correcting for the large offset acceptance, the source is detected at the $5.7 \sigma \mathrm{CL}$ in $48.4 \mathrm{~h}$. $1 \mathrm{ES} 1312-423$ was found to be very faint in the VHE range, with a flux of $\sim 0.5 \%$ of the Crab flux, making it one the faintest VHE source currently known. Contemporaneous multi-wavelength data from radio to high energies with ATCA, ATOM, Swift and Fermi/LAT were used to study its spectral energy distribution, which can be well described using a one-zone SSC model.

AP Lib, a low-frequency-peaked BL Lac object, was discovered as a VHE $\gamma$-ray 
emitter with H.E.S.S. at $7 \sigma$ CL in $11 \mathrm{~h} \cdot \sqrt[36]{6}$ From Fermi/LAT data analysis, it was found that this object also exhibits a hard $\gamma$-ray spectrum at high energy with $\Gamma=2.1 \pm 0.1$ in the LAT energy range. Together with contemporaneous Swift and RXTE data, the spectral energy distribution of AP Lib displays an atypical, unusually broad component at high energy, with respect to other VHE emitters (see Fig. (4). Such a component seriously challenges simple SSC scenarios, and multiple

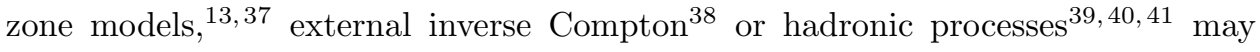
have to be invoked to interpret its multi-wavelength emission.

\subsection{Extragalactic background light}

The properties of the extragalactic background light ${ }^{42}$ (EBL), the sum of the light emitted by stars and galaxies and its reprocessing by dust, have long been studied both using direct - in the infrared and optical bands - and indirect measurements. The VHE photons emitted in a source interact with the EBL field, softening the intrinsic VHE spectrum as observed from the Earth. By accumulating statistics from the brightest blazars detected with H.E.S.S., the global imprint of the EBL in VHE $\gamma$-ray observations has been significantly revealed at the $8.8 \sigma \mathrm{CL}^{43}$ (see Fig. 5). The Fermi/LAT collaboration also detected such an imprint at high energies for more distant blazars, $\stackrel{44}{a}$ up to $z \simeq 1.6$.

\section{Conclusions}

The recent H.E.S.S. results on VHE extragalactic sources show a broad diversity, with e.g. flux upper limits obtained both in the prompt and afterglow phases of GRB 060602B, constraining limits put on galaxy cluster emission models, as well as the need for simultaneous multi-wavelength studies of AGN to better understand the physics at the sources, and last but not least the detection of the EBL imprint on blazar spectra.

With the ramp-up of the fifth H.E.S.S. telescope, more results are expected to come soon, both in monoscopic mode with events triggering the biggest telescope alone, as well as hybrid events that trigger it and at least another telescope in the array. Those heterogeneous data taking capabilities will ensure both an energy threshold as low as possible, and a better sensitivity in the overall energy range. In this regard, distant cosmological sources could be observed with H.E.S.S., to better understand both the intrinsic emission at work and further investigation of properties of the EBL. The energy coverage will also help investigating the radiative processes in AGN, and specifically FSRQ, by probing the high energy peak of their spectra. In a further future, the $\mathrm{CTA}^{45}$ will help widening our views on the VHE emitting Universe.

\section{Acknowledgments}

I would like to thank the conference organisers for the opportunity to speak at the $4^{\text {th }}$ High Energy Phenomena in Relativistic Outflows (HEPRO IV) meeting, and 


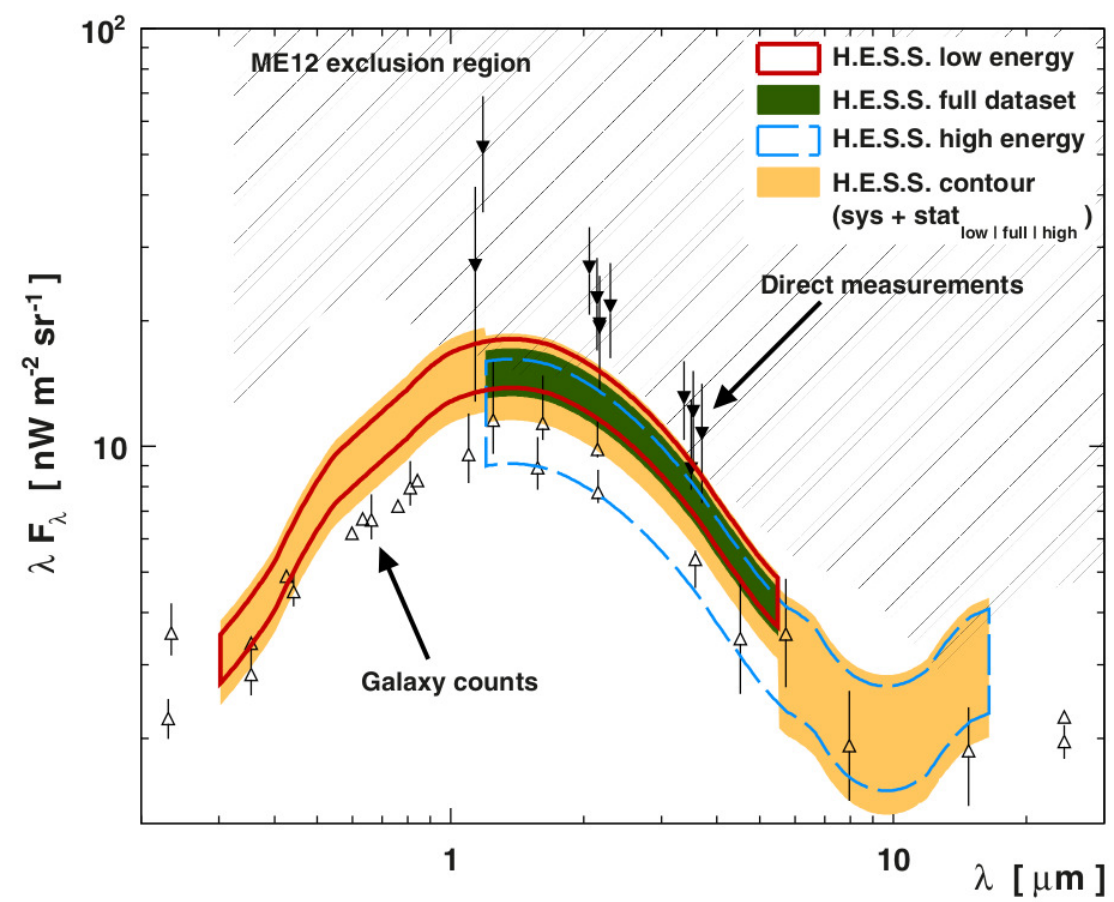

Fig. 5. Detection of the EBL with H.E.S.S. See Ref. 43 for more details.

for organising an interesting and enjoyable conference.

The support of the Namibian authorities and of the University of Namibia in facilitating the construction and operation of H.E.S.S. is gratefully acknowledged, as is the support by the German Ministry for Education and Research (BMBF), the Max Planck Society, the German Research Foundation (DFG), the French Ministry for Research, the CNRS-IN2P3 and the Astroparticle Interdisciplinary Programme of the CNRS, the UK Science and Technology Facilities Council (STFC), the IPNP of the Charles University, the Czech Science Foundation, the Polish Ministry of Science and Higher Education, the South African Department of Science and Technology and National Research Foundation, and by the University of Namibia. We appreciate the excellent work of the technical support staff in Berlin, Durham, Hamburg, Heidelberg, Palaiseau, Paris, Saclay, and in Namibia in the construction and operation of the equipment.

\section{References}

1. H.E.S.S. Collaboration, ApJ 757, 158 (2012).

2. MAGIC Collaboration et al., A $\xi A$ 539, L2 (2012).

3. F. Aharonian, A. Akhperjanian, M. Beilicke et al., A\&A 403, L1 (2003).

4. F. Aharonian, A. G. Akhperjanian, A. R. Bazer-Bachi et al., Science 314, 1424 (2006).

5. F. Aharonian, A. G. Akhperjanian, G. Anton et al., ApJ 695, L40 (2009). 
6. M. Ackermann, M. Ajello, A. Allafort et al., ApJ 743, 171 (2011).

7. MAGIC Collaboration et al., arXiv:1305.5147 (2013).

8. VERITAS Collaboration, VLBA 43 GHz M 87 Monitoring Team, H.E.S.S. Collaboration, MAGIC Collaboration, Science 325, 444 (2009).

9. H.E.S.S. Collaboration, MAGIC Collaboration, VERITAS Collaboration et al., ApJ 746, 151 (2012).

10. Fermi/LAT Collaboration, Science 328, 725 (2010).

11. A. A. Abdo, M. Ackermann, M. Ajello et al., ApJ 719, 1433 (2010).

12. M. Chiaberge, A. Celotti, A. Capetti \& G. Ghisellini, A $\& A$ 358, 104 (2000).

13. J.-P. Lenain, C. Boisson, H. Sol \& K. Katarzyński, A\&A 478, 111 (2008).

14. M. Georganopoulos \& D. Kazanas, ApJ 594, L27 (2003).

15. F. M.Rieger \& F. A. Aharonian, $A \& A$ A 506, L41 (2009).

16. N. Sahakyan, R. Yang, F. A. Aharonian \& F. M. Rieger, ApJ 770, L6 (2013).

17. M. Ackermann, M. Ajello, K. Asano et al., arXiv:1303. 2908 (2013).

18. P. Mészáros, Astroparticle Physics 43, 134 (2013).

19. S. Inoue, J. Granot, P. T. O'Brien et al. (for the CTA Consortium), Astroparticle Physics 43, 252 (2013).

20. F. Aharonian, A. G. Akhperjanian, U. Barres DeAlmeida et al., ApJ 690, 1068 (2009).

21. R. Wijnands, E. Rol, E. Cackett, R. L. C. Starling \& R. A. Remillard, MNRAS 393, 126 (2009).

22. B. R. McNamara \& P. E. J. Nulsen, ARA\&A 45, 117 (2007).

23. M. W. Wise, B. R. McNamara, P. E. J. Nulsen, J. C. Houck \& L. P. David, ApJ 659, 1153 (2007).

24. H.E.S.S. Collaboration et al., A\&A 545, A103 (2012).

25. MAGIC Collaboration et al., ApJ 730, L8 (2011).

26. MAGIC Collaboration, Science 320, 1752 (2008).

27. H.E.S.S. Collaboration, A $\& A$ 554, A107 (2013).

28. F. Aharonian, A. G. Akhperjanian, A. R. Bazer-Bachi et al., Nature 440, 1018 (2006).

29. G. Fossati, L. Maraschi, A. Celotti, A. Comastri \& G. Ghisellini, MNRAS 299, 433 (1998).

30. H.E.S.S. Collaboration et al., A\&A 552, A118 (2013).

31. N. Craig \& A. Fruscione, $A J$ 114, 1356 (1997).

32. E. S. Perlman, P. Padovani, P. Giommi et al., AJ 115, 1253 (1998).

33. H. Landt, MNRAS 423, L84 (2012).

34. S. Pita, P. Goldoni, C. Boisson et al., in American Institute of Physics Conference Series, eds. F. A. Aharonian, W. Hofmann \& F. M. Rieger, 1505, 566 (2012).

35. H.E.S.S. Collaboration et al., MNRAS 434, 1889 (2013).

36. P. Fortin, S. Fegan, D. Horan et al. (for the Fermi/LAT and H.E.S.S. Collaborations), in 25th Texas Symposium on Relativistic Astrophysics, 199 (2010).

37. F. Tavecchio \& G. Ghisellini, MNRAS 385, L98 (2008).

38. M. Sikora \& M. Zbyszewska, MNRAS 212, 553 (1985).

39. K. Mannheim, P. L. Biermann \& W. M. Kruells, A\& A 251, 723 (1991).

40. F. A. Aharonian, New Astronomy 5, 377 (2000).

41. A. Mücke \& R. J. Protheroe, Astroparticle Physics 15, 121 (2001).

42. M. G. Hauser \& E. Dwek, ARA\&A 39, 249 (2001).

43. H.E.S.S. Collaboration, A\&A 550, A4 (2013).

44. Fermi/LAT Collaboration, Science 3381190 (2012).

45. CTA Consortium, Astroparticle Physics 43, 3 (2013). 